\title{
Necrotizing Pneumonia In Infant: Rare Complication of Community Acquired Pneumonia
}

\author{
Adhikari SB' ${ }^{1}$ Chaudhary RP²
}

\begin{abstract}
Necrotizing pneumonia, rare complication of Communityacquired bacterial pneumonia (CABP), bear its own morbidity and mortality. Even though CABP generally respond well to the antibiotic treatment, but complications like empyema, necrotizing pneumonia with pneumatocoel formation (cavitary necrosis) supervene in mainly immune-compromised patient. Although cavitary necrosis is manifested as a severe disease, most children show complete recovery even without surgical treatment and have normal chest radiographs at long term. Some cases may required surgical intervention like tube thoracotomy or open thoracotomy. A case is presented of an infant that developed necrotizing pneumonia with pneumatocoel formation during treatment of bacterial pneumonia. Tube thoracotomy was performed due to conservative treatment failure.
\end{abstract}

\section{Introduction}

No ecrotizing pneumonia (NP) is quite uncommon in children compare with adult age group, rare complication of pneumonia. It is characterized by progressive pneumonic illness in a previously healthy child despite appropriate antibiotic therapy, and runs a protracted clinical course ${ }^{1}$. NP lies on a spectrum between pulmonary abscess and pulmonary gangrene, and is accompanied frequently by empyema and bronchopleural fistulae (BPF). It is speculated that reduced blood flow from thrombosed vessels decreases antibiotic concentrations within the affected lung tissue, leading to persistent infection and further destruction of pulmonary tissue ${ }^{2}$.

NP may be complicating $0.8-7 \%$ of all cases of communityacquired pneumonia $(\mathrm{CAP})^{3}$, although studies retrospectively evaluating the incidence have found it to be increasing during the past 20 years. The most common pathogens associated with NP in children are pneumococci and $S$. aureus.

This rare case reporting so far is the first of its kind published from Nepal. Research to address challenges in the aetiological diagnosis of pneumonia and widespread implementation of treatment interventions (antibiotic) and dealing with complications (tube thoracotomy or thoracotomy) are necessary to mitigate the burden of pneumonia and improve child survival.
${ }^{1}$ Dr. Suman Bikram Adhikari, MBBS, MS, ${ }^{2}$ Dr. Ramananda Prasad Chaudhary, MBBS. MS. FICS Paed, Fellow Paediatric Urology, Professor, Department of Paediatric Surgery. Both from Ishan Children and Maternity Hospital, Kathmandu, Nepal.

Address for correspondence

Dr. Suman Bikram Adhikari

E-mail: sumanchetri@hotmail.com

\section{How to cite}

Adhikari SB, Chaudhary RP. Necrotizing Pneumonia In Infant: Rare Complication of Community Acquired Pneumonia. J Nepal Paediatr Soc 2018;38(2):135-139.

doi:http://dx.doi.org/10.3126/jnps.v38i2.21327

This work is licensed under a Creative Commons Attribution 3.0 License. 


\section{The Case}

Ten months male infant from remote village area presented to local hospital with shortness of breath and fever for few days. He was treated as community based bacterial pneumonia with intravenous antibiotics. But patient condition did not improve and Chest radiography showed cavitary lesion in upper zone of right lung suspecting congenital lobar emphysema. Patient was then referred to paediatric surgeon for further management. On admission, the child was conscious, not-exhausted, low grade fever with occasional dyspnoeic. Physical findings: pulse 120/min, respiration $40 / \mathrm{min}$, body temperature $38.4^{\circ} \mathrm{C}$, and $\mathrm{O}_{2}$ saturation in room air $97 \%$. There was dullness on percussion over the right upper and middle zone. There was decreased breath sound in right upper zone with crepitation at right lower zone with normal breath sound in left hemi-thorax.

Laboratory findings: Total Leukocytes: $25.6 \times 10^{9} /$ litre, of which neutrophils: $50 \%$, lymphocytes: $46 \%$ and monocytes: 2\%, Haemoglobin $9.3 \mathrm{mg} / \mathrm{dl}$, Platelets: 545x10\%/litre, and C-reactive protein (CRP): $1.2 \mathrm{mg} / \mathrm{dl}$.

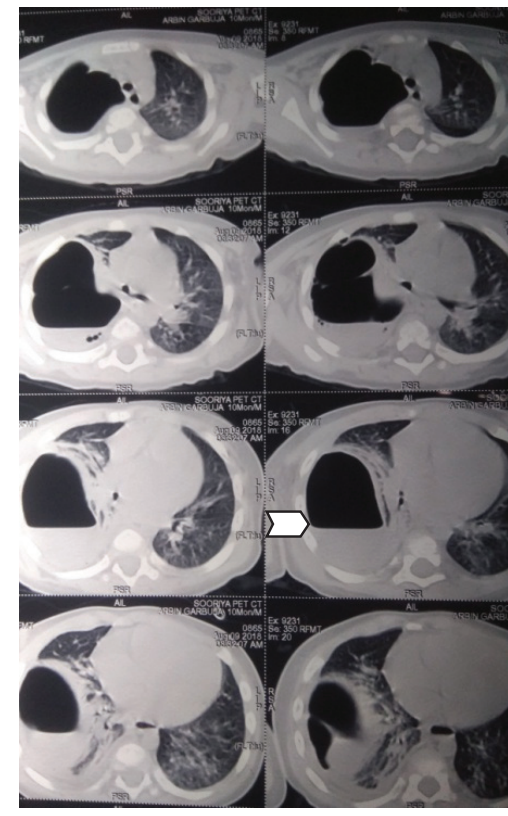

Fig. 1: CECT chest shows cavity with air fluid level (Chevron).

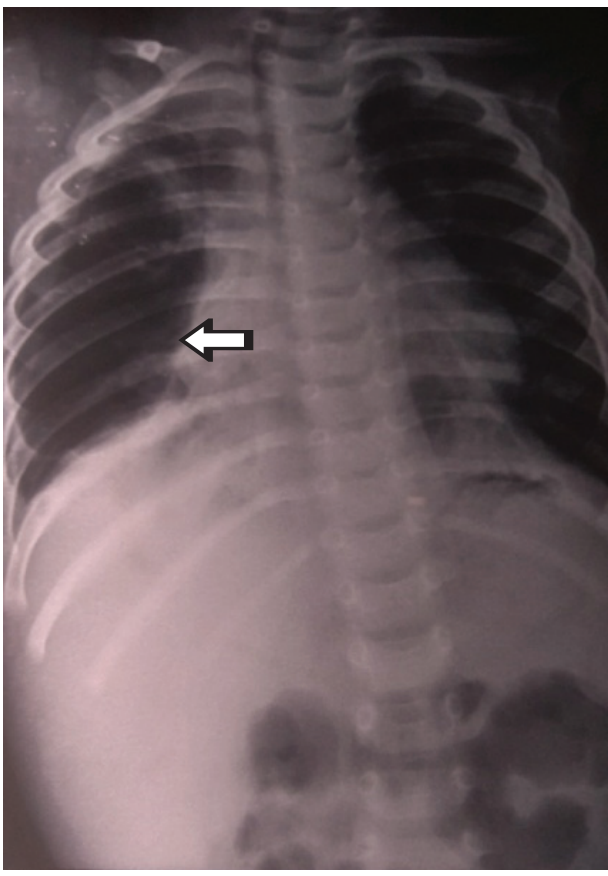

Fig 3: Showing the Chest $x$-ray with air filled cavity at right hemi-thorax (Arrow)

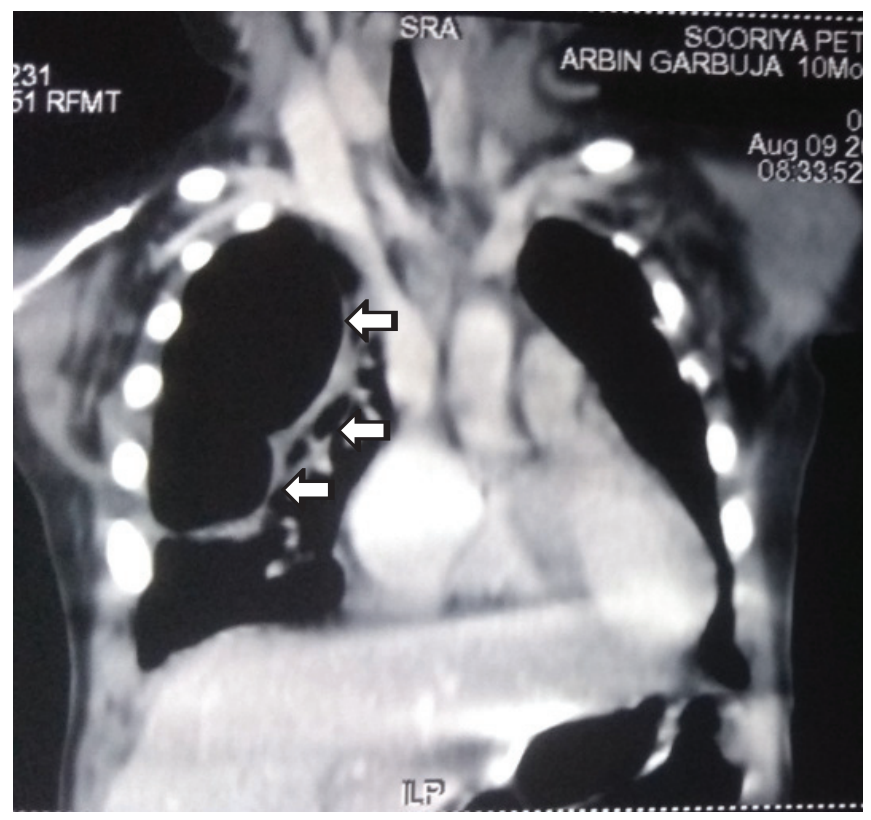

Fig. 2: CECT chest shows multiloculated cavities at right hemithorax (arrow).

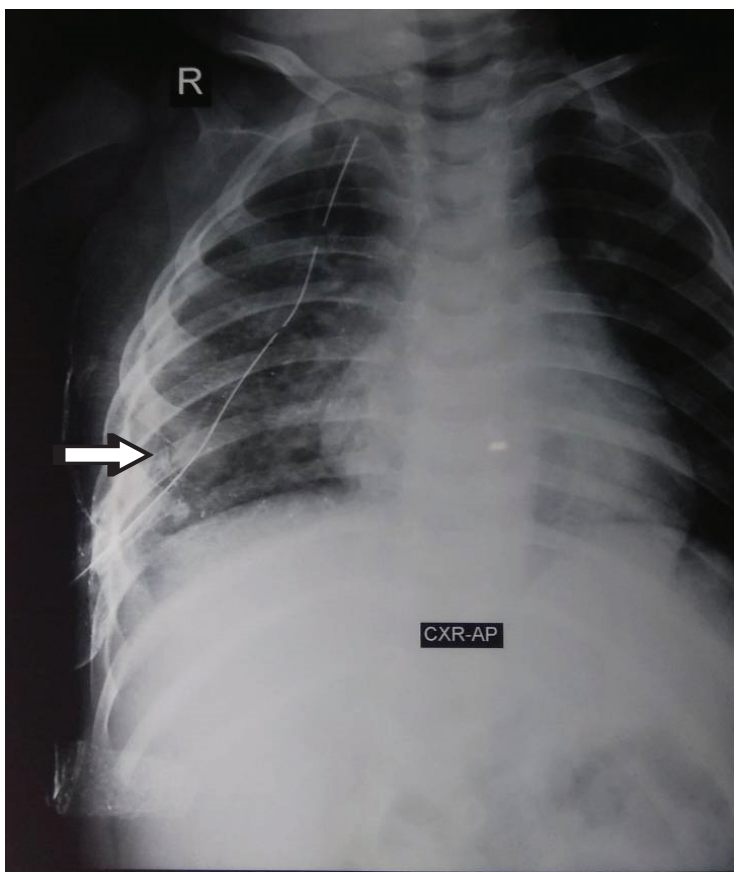

Fig 4: Showing obliteration of cavity with right lung expansion with minimal consolidation remnant (Arrow) 
CECT Chest was suggested which revealed large cystic cavity lesion measuring $5.5 \times 6.2 \times 7.7 \mathrm{~cm}$ with internal air fluid level noted involving right upper lobe likely sequelae of necrotizing pneumonia. We performed right tube thoracotomy supplemented by intravenous antibiotics. Culture report revealed no organism growth. She recovered well and repeat chest x-rays showed adequate chest expansion with some residual pneumonic consolidation for which oral antibiotics was prescribed.

\section{Discussion}

Most children with NP are young, aged $<5$ years, and have been healthy previously. The clinical features are those of pneumonia with fever, cough, chest pain, tachypnoea, and localizing chest signs that may include percussion dullness, decreased breath sounds, and/ or bronchial breathing. Symptoms may be present for several days before presentation and despite receiving appropriate therapy, the children are often disproportionately sick with persistent fever, respiratory distress and clinical and/or radiographic signs of a nonresponding or progressive pneumonia ${ }^{4}$.

The major pathogens are S. pneumoniae and $S$. aureus and the diagnosis should be considered when, despite appropriate antibiotics, the child remains febrile and unwell with persistent signs of respiratory distress and pneumonia. Other respiratory bacterial and fungal pathogens reported occasionally in studies included in the present review are Streptococcus pyogenes, $S$. anginosus group, Haemophilus influenza, Pseudomonas aeruginosa, Stenotrophomonas maltophilia and anaerobic organism. Viruses are rarely the sole cause of NP.

The diagnosis of NP should always be considered in a child with pneumonia who remains unwell, despite at least $72 \mathrm{~h}$ of appropriate antibiotics. Occasionally children with NP can deteriorate rapidly following their initial presentation with features of severe sepsis, including septic shock, multi-organ failure, and hypoxic respiratory failure ${ }^{5}$. The diagnosis of NP is generally made by chest imaging studies. Although chest radiographs will demonstrate the underlying pneumonia, accompanying empyema, and if there is a resulting mediastinal shift, this investigation is only diagnostic in $27-41 \%$ of children with NP where one or more small lucencies or pneumatocoels are seen at a median of 4-8 days following hospitalization ${ }^{6,7}$. The poor sensitivity of chest radiographs results from the cavitary lesions being filled with fluid following liquefaction necrosis that has the same density as the adjacent consolidated lung.

Contrast-enhanced chest CT scans are more sensitive than chest radiographs and have become the standard imaging procedure for making the diagnosis of NP, evaluating the lung parenchyma changes not visible on plain chest radiographs, and determining whether any underlying congenital lung malformations exist ${ }^{8}$. The key diagnostic features are poor or absent vascularity, loss of pulmonary architecture, and finally cavity formation.

Histopathological findings in autopsy and surgical lung specimens from adults and children with NP are characterized by necrosis of lung parenchyma, which was thought primarily to be a vascular process triggered by infection leading to vasculitis, activation of the coagulation system and thrombotic occlusion of intrapulmonary vessels accompanied by cavity formation ${ }^{2,8,9}$. The mixture of coagulation and liquefactive lung necrosis leads to one or more thin-walled cavities that can form pneumatocoels from the one-way passage of gas ${ }^{10}$, or evolve into pulmonary abscesses ${ }^{8}$.

A multi-disciplinary team of paediatric respiratory physicians, paediatric or thoracic surgeons, and infectious diseases experts is often required. The overarching aims are to control and ultimately reverse the patho-biologic changes associated with NP. These include providing supplemental oxygen to relieve hypoxia, ensuring adequate analgesia to reduce pleuritic pain and improve ventilation, administering prolonged antibiotic therapy, and decreasing any mass effect or intrathoracic pressure by draining gas and/or intra-pleural fluid $8,9,11$. Correcting fluid and electrolyte abnormalities and attention to nutrition, including managing hypoalbuminemia, is also important. Some children will require circulatory and ventilation support, while occasionally extracorporeal membrane oxygenation (ECMO) is used in those with refractory hypoxemic respiratory failure ${ }^{12}$.

A prolonged course of IV antibiotics is the cornerstone of therapy. The initial choice of antibiotics in otherwise healthy, fully immunized children should be the same as for empyema and cover gram positive organisms, especially pneumococci, $S$. aureus and $S$. pyogenes ${ }^{13}$, taking into account local epidemiologic and microbiologic data. Treatment can then be tailored according to microbiological results, although these may only be positive in $8-55 \%$ of cases. The optimal duration for antibiotic treatment of NP is unknown. The median length of antibiotic courses range from 13 to 42 days, with 3 of the 5 studies providing these data reporting a median antibiotic course duration of 28 days $^{3}$. Switching from IV to oral antibiotics is appropriate once the child is afebrile for at least $24 \mathrm{~h}$ and no longer showing signs of sepsis, their respiratory distress is resolving, enteral 
feeds are being tolerated, and inflammatory markers are declining ${ }^{[14]}$. At this point antibiotics are continued for at least another 10-14 days, a recommendation that aligns with consensus guidelines for paediatric CAP complications $^{13}$.

While it is recommended that surgical intervention is kept to a minimum to avoid risking BPF ${ }^{[3]}$, this is not always possible if a large pyo-pneumothorax, tension pneumatocoel and/or loculated empyema are already present, especially if they persist or lead to mass effects causing hemodynamic instability and further compromise of ventilation. Chest tube drainage alone for large pyopneumothorax may suffice, but for those with loculated empyema, chest tube insertion and installation of intra-pleural fibrinolytic is an option preferred by some experts $^{11}$. However, chest tube drainage $>7$ days and fibrinolytic may also be associated with increased risk of BPF developing ${ }^{3}$. Surgical intervention with either video-assisted thoracoscopic surgery or minithoracotomy to debride pyogenic material around the lung (decortication), breakdown loculations, and remove pus may be required when fever, signs of sepsis and/ or respiratory distress continue, despite chest tube insertion with or without fibrinolytic therapy and repeat imaging shows persistent or increasing intra-pleural collections ${ }^{13}$. However, if pleural disease is minimal, the aforementioned symptoms may be from the underlying NP and continuing IV antibiotics without surgery is recommended. The next aim of surgery is managing progressive lung parenchymal necrosis. This involves segmental or lobar resection or pneumonectomy and is rarely required in children.
Children with NP usually have a prolonged hospital stay. The median length of stay in ranged from 12 to 30 days. Not surprisingly, those undergoing surgery had a longer stay in hospital than those managed conservatively ${ }^{[15]}$. Unlike adults where case fatality rates for NP can range from approximately $40-50 \%{ }^{16}$, deaths in children are uncommon despite them often being critically ill and requiring management in intensive care units.

Until there is a greater understanding of why NP occasionally complicates pneumonia, its prevention will depend upon reducing CAP and its severity. Decreases in CAP are attributed to a combination of improved housing, water supply and hygiene, better indoor air quality, reduced parental tobacco smoking, increased education, breastfeeding rates and nutrition, and greater healthcare access and vaccine uptake ${ }^{17}$. Vaccines contributing to this success globally are pertussis, measles, Hib, and pneumococcal conjugate vaccines ${ }^{18}$.

\section{Conclusion}

Despite substantial increases in our understanding of the clinical syndrome of pneumonia and its aetiology, its accurate diagnosis and sequelae are challenging when only clinical indicators are relied up on. Diagnosis improves modestly with addition of laboratory, microbiological, or radiological investigations. Children may be at risk of misdiagnosis and inadequate treatment leading to grave complications like Necrotizing Pneumonia.

5. Schwartz KL, Nourse C. Panton-valentine leucocidinassociated Staphylococcus aureusnecrotizing pneumonia in infants: a report of four cases and review of the literature. Eur J Pediatr 2012;171:71117 Available at; https://pdfs.semanticscholar.org/fbd1/ c05f2a8599d8733c4ad6162ab6aa19a02549.pdf

6. Lemaitre C, Angoulvant F, Gabor F, Makhoul J, Bonacorsi S, Naudin J, et al. Necrotizing pneumonia in children. Report of 41 cases between 2006 and 2011 in a French tertiary care center. Pediatr Infect Dis J 2013;32:1146-49. DOI: 10.1097/ INF.0b013e31829be1bb

7. Donnelly LF, Klosterman LA. Cavitatory necrosis complicating pneumonia in children: sequential findings on chest radiography. Am J Roentgenol 1998;171:25356. DOI: 10.2214/ajr.171.1.9648799

8. Thomas MF, Wort A, Spencer DA. Management and complications of pneumonia. Paediatr Child Health 2014;25:172-78. DOI: doi.org/10.1016/j. paed.2014.11.004 
9. Loizzi M, De Palma A, Pagliarulo V, Loizzi D, Sollitto F. Pulmonary infections of surgical interest in childhood. Thorac Surg Clin 2012;22:387-401. DOI: 10.1016/j. thorsurg.2012.04.005.

10. Cicak B, Verona E, Mihatov-Stefanovic I. Necrotizing pneumonia in infants. Acta Clin Croat. 2010;49:32126.

11. Spencer DA, Thomas MF. Necrotising pneumonia in children. Paediatr Respir Rev 2014;15:240-5. DOI: 10.1016/j.prrv.2013.10.001.

12. Stroud MH, Okhuysen-Cawley R, Jaquiss R, Berlinski A, Fiser RT. Successful use of extracorporeal membrane oxygenation in severe necrotizing pneumonia caused by Staphylococcus aureus. Pediatr Crit Care Med 2007;8:282-87. DOI: 10.1097/01. PCC.0000262795.11598.56

13. Islam $\mathrm{S}$, Calkins $\mathrm{CM}$, Goldin $\mathrm{AB}$, et al. The diagnosis and management of empyema in children: a comprehensive review from the APSA outcomes and clinical trial committee. J Pediatr Surg 2012;47:210110. DOI: 10.1016/j.jpedsurg.2012.07.047.
14. McMullan BJ, Andresen D, Blyth CC, et al. Antibiotic duration and the timing of the switch from intravenous to oral route for bacterial infections in children: systematic review and guidelines. Lancet Infect Dis 2016;16:e13952. DOI: 10.1016/S1473-3099(16)30024-X

15. Lai JY, Yang W, Ming YC. Surgical management of complicated necrotizing pneumonia in children. Pediatr Neonatol 2017;58(4):321-327. DOI: 10.1016/j. pedneo.2016.06.002.

16. Li HT, Zhang TT, Huang J, Zhou YQ, Zhu JX, Wu $B Q$. Factors associated with the outcome of life threatening necrotizing pneumonia due to communityacquired Staphylococcus aureus in adult and adolescent patients. Respiration 2011;81:448-60. DOI: 10.1159/000319557.

17. Izadnegahdar R, Cohen AL, Klugman KP, Qazi SA. Childhood pneumonia in developing countries. Lancet Respir Med 2013;1:574-84. DOI: 10.1016/S22132600(13)70075-4.

18. Williams DJ, Shah SS. Community-acquired pneumonia in the conjugate vaccine era. J Pediatr Infect Dis Soc 2012;1:314-28. 